\title{
Multi-constellation single-frequency ionospheric-free precise point positioning with low-cost receivers
}

\author{
Jacek Paziewski ${ }^{1}$ (D) \\ Received: 19 April 2021 / Accepted: 26 November 2021 / Published online: 9 December 2021 \\ (c) The Author(s) 2021
}

\begin{abstract}
We analyze the observation quality, assess the performance and identify the constraints of quadruple-constellation singlefrequency ionospheric-free precise point positioning (SF-IF PPP) with low-cost receivers. It is revealed that low-cost receivers with patch antennas exhibit lower $\mathrm{C} / \mathrm{N} 0$ records and a weaker elevation dependence of $\mathrm{C} / \mathrm{N} 0$ than the high-grade equipment. The results demonstrate that low-cost receivers can offer code measurements with similar noise compared to high-grade receivers providing that the multipath effect is eliminated. Regarding positioning performance, it is shown how SF-IF PPP for the high-grade receiver converges approximately two times faster than for the low-cost receiver with a patch antenna. It is confirmed that an application of a survey-grade antenna instead of the patch one noticeably enhances the performance of low-cost receiver SF-IF PPP. The study also reveals that the multipath effect is a dominant factor that constrains the performance of SF-IF PPP with low-cost receivers.
\end{abstract}

Keywords Low-cost receivers $\cdot$ Precise point positioning $\cdot$ GNSS observation assessment $\cdot$ Multipath

\section{Introduction and motivation}

An increasing interest in the precise positioning with lowcost receivers is recognized. In one of the first research in this area, Wisniewski et al. (2013) assessed the feasibility of precise positioning with the LEA-6T low-cost receiver. This was followed by the studies on single-baseline realtime kinematic (RTK) by Odolinski and Teunissen (2016, 2017), who investigated positioning with dual-system single-frequency (SF) and dual-frequency (DF) observations, respectively, and Garrido-Carretero et al. (2019), who showed the performance of SF-RTK with u-blox NEO-M8P. A centimeter-level positioning of multi-constellation RTK with Skytraq S2525F8-BD, u-blox ZED F9P and EVK-M8T receivers during high ionospheric activity and for long-baselines was reported in (Odolinski and Teunissen 2019, 2020), respectively.

However, there is still a need to evaluate the accuracy level that may be reached with low-cost receivers in singlefrequency precise point positioning (SF-PPP) mode. This is

Jacek Paziewski

jacek.paziewski@uwm.edu.pl

1 University of Warmia and Mazury in Olsztyn, Oczapowskiego 1, 10-719 Olsztyn, Poland warranted by a high demand for autonomous positioning and the fact that many devices are equipped with GNSS chipsets that offer observations on a single-frequency band only.

The most important challenge for SF-PPP is the elimination of the ionospheric delay. This error may be handled by using ionospheric models and global ionospheric maps. Respective research on this topic for low-cost receiver SFPPP is found in Gill et al. (2017), use of real-time ionospheric products provided, e.g., by the Centre National d'Etudes Spatiales is found in Wang et al. (2020), and models such as ionospheric-weighted, ionospheric-constrained, and ionospheric-free (IF) were used in Li et al. (2019). The recently developed Smart-PPP for smartphone observations was addressed in Wang et al. (2021). Single-frequency ionospheric-corrected code observations of low-cost receivers were used in PPP by Nie et al. (2019). However, the method proposed by the authors combines SF measurements with conventional IF-DF linear combination (LC) and thus is suitable for DF receivers only.

Another option for handling ionospheric delay in SF positioning is to employ a combination of phase and code observations that was originally known as GRoup And PHase Ionospheric Correction (GRAPHIC) (Yunck 1996). The quality of code observations is a dominant factor that drives the positioning performance based on this linear combination. 
Therefore, a growing precision of code pseudoranges offered by modernized and emerging constellations is one of the aspects that induced us to draw special attention to this single-frequency ionospheric-free PPP model called SF-IF PPP. SF-IF PPP model was, until now, applied only to the observations of selected constellations, e.g., GPS + GLONASS as shown in (Cai et al. 2013), GPS + Galileo that was analyzed in (Bahadur and Nohutcu 2020) or single constellation, e.g., BDS (Wanninger and Beer 2015). Moreover, existing studies were based on the observations collected by high-grade receivers and antennas and with such equipment a decimeter- to sub-decimeter-level accuracy depending on the session length was reported (Bahadur and Nohutcu 2021).

Such progress motivates to assess, for the first time, the performance of quadruple-constellation SF-IF PPP of the low-cost receivers against high-grade ones and to identify the constraints of SF-PPP with low-cost receivers. An insight into the quality of GNSS observations of current low-cost receivers is provided. Furthermore, the observation quality and positioning results are analyzed in the light of the multipath impact, and the potential benefit from applying a survey-grade antenna instead of a low-cost one is verified.

In the next section, the functional model of SF-IF PPP is presented. The following section describes the data collection and the experiment design. Next, the observation quality analysis is given. Afterward, the performance of low-cost receiver SF-IF PPP against that of a high-grade receiver is assessed, and the impact of the multipath effect on the positioning is investigated. Finally, the conclusions are provided in the last section.

\section{Methodology of single-frequency ionospheric-free precise point positioning}

The observation equations for phase $\Phi$ and code $P$ undifferenced signals between the satellite $s$ and receiver $r$, and generalized to frequency $i$ read:

$P_{r, i}^{s}=\rho_{r, 0}^{s}+c \cdot\left(\mathrm{d} t_{r}-\mathrm{d} t^{s}\right)+I_{r, i}^{s}+T_{r}^{s}+b_{r, i}-b_{i}^{s}+\epsilon_{r, P}^{s}$

$\Phi_{r, i}^{s}=\rho_{r, 0}^{s}+c \cdot\left(\mathrm{d} t_{r}-\mathrm{d} t^{s}\right)-I_{r, i}^{s}+T_{r}^{s}+\lambda_{i} \cdot N_{r, i}^{s}+B_{r, i}-B_{i}^{s}+\epsilon_{r, \Phi}^{s}$

in which $\rho_{r, 0}^{s}$ denotes the geometric range between the satellite and a priori position of the station in meters; $c$ represents the speed of light in meters per second; $\mathrm{d} t$ and $\mathrm{d} t^{s}$ correspond to the receiver and satellite clock corrections in seconds, respectively; $\lambda_{i}$ is the signal wavelength in meters on selected frequency $i ; I_{r, i}^{s}$ and $T_{r}^{s}$ are the slant ionospheric and tropospheric delays in meters, respectively; $N_{r, i}^{s}$ is the integer ambiguity of the phase observable in cycles; $B_{r, i}$ and $B_{i}^{s}$ are the receiver and satellite phase delays in meters, while $b_{r, i}$ and $b_{i}^{s}$ correspond to the receiver and satellite code pseudorange delays in meters, respectively; finally, $\epsilon_{r, P}^{s}$ and $\epsilon_{r, \Phi}^{s}$ denote the noise of code and phase observations, correspondingly.

The availability of SF phase and code observations creates an opportunity to employ GRAPHIC IF-LC. The combination takes advantage of the fact that phase and code pseudorange observations transmitted on the same frequency band are subject to an ionospheric delay of the same magnitude but the opposite sign. However, it may also cause a model rank deficiency with single-epoch observations when using a least-squares adjustment (Sterle et al. 2015). The GRAPHIC combination applied to undifferenced observations is expressed as follows:

$$
\begin{gathered}
\left(P_{r, i}^{s}+\Phi_{r, i}^{s}\right) / 2-\rho_{r, 0}^{s}+c \cdot \mathrm{d} t^{s}-m h_{r}^{s} \cdot \mathrm{ZHD}_{r}+b_{i}^{s} / 2 \\
\quad=e_{r}^{s} \cdot \delta X_{r}+c \cdot \mathrm{d} \bar{t}_{r}+m w_{r}^{s} \cdot \mathrm{ZWD}_{r}+A_{r}^{s}+\epsilon_{r, \mathrm{SF}-\mathrm{IF}}^{s}
\end{gathered}
$$

where $m h_{r}^{s}$ and $m w_{r}^{s}$ are coefficients of the hydrostatic and non-hydrostatic tropospheric mapping functions, respectively; $\mathrm{ZHD}_{r}$ and $\mathrm{ZWD}_{r}$ represent zenith hydrostatic and non-hydrostatic tropospheric delays; $e_{r}^{s}$ denotes the line-ofsight vector from the user receiver to the satellite; $\delta X_{r}$ refers to the vector of the corrections to a priori coordinates of the station in meters; $\mathrm{d} \bar{t}_{r}$ is the receiver clock offset that combines receiver clock corrections and the temporally variable receiver hardware delays in seconds, $A_{r}^{s}$ is the non-integer ambiguity term that aggregates one half of the phase integer ambiguity and satellite-specific but constant biases. Finally, $\epsilon_{r, \mathrm{SF}-\mathrm{IF}}^{s}$ denotes observational noise of SF-IF LC.

The final system of observation equations for combined quadruple-constellation signals that handles different for each system time scales reads:

$$
\begin{aligned}
& \left(P_{r, i}^{s, G}+\Phi_{r, i}^{s, G}\right) / 2-\rho_{r, 0}^{s, G}+c \cdot \mathrm{d} t^{s, G}-m h_{r}^{s, G} \cdot \mathrm{ZHD}_{r}+b_{i}^{s, G} / 2 \\
& \quad=e_{r}^{s, G} \cdot \delta X_{r}+c \cdot \mathrm{d} \bar{t}_{r}+m w_{r}^{s, G} \cdot \mathrm{ZWD}_{r}+A_{r}^{s, G}+\epsilon_{r, \mathrm{SF}-\mathrm{IF}}^{s, G} \\
& \left(P_{r, i}^{s, R}+\Phi_{r, i}^{s, R}\right) / 2-\rho_{r, 0}^{s, R}+c \cdot \mathrm{d} t^{s, R}-m h_{r}^{s, R} \cdot \mathrm{ZHD}_{r}+b_{i}^{s, R} / 2 \\
& \quad=e_{r}^{s, R} \cdot \delta X_{r}+c \cdot\left(\mathrm{d} \bar{t}_{r}+\mathrm{d} t_{r}^{R-G}\right)+m w_{r}^{s, R} \cdot \mathrm{ZWD}_{r}+A_{r}^{s, R}+\epsilon_{r, \mathrm{SF}-\mathrm{IF}}^{s, R} \\
& \left(P_{r, i}^{s, E}+\Phi_{r, i}^{s, E}\right) / 2-\rho_{r, 0}^{s, E}+c \cdot \mathrm{d} t^{s, E}-m h_{r}^{s, E} \cdot \mathrm{ZHD}_{r}+b_{i}^{s, E} / 2 \\
& \quad=e_{r}^{s, E} \cdot \delta X_{r}+c \cdot\left(\mathrm{d} \bar{t}_{r}+\mathrm{d} t_{r}^{E-G}\right)+m w_{r}^{s, E} \cdot \mathrm{ZWD}_{r}+A_{r}^{s, E}+\epsilon_{r, \mathrm{SF}-\mathrm{IF}}^{s, E} \\
& \left(P_{r, i}^{s, C}+\Phi_{r, i}^{s, C}\right) / 2-\rho_{r, 0}^{s, C}+c \cdot \mathrm{d} t^{s, C}-m h_{r}^{s, C} \cdot \mathrm{ZHD}_{r}+b_{i}^{s, C} / 2 \\
& \quad=e_{r}^{s, C} \cdot \delta X_{r}+c \cdot\left(\mathrm{d} \bar{t}_{r}+\mathrm{d} t_{r}^{C-G}\right)+m w_{r}^{s, C} \cdot \mathrm{ZWD}_{r}+A_{r}^{s, C}+\epsilon_{r, \mathrm{SF}-\mathrm{IF}}^{s, C}
\end{aligned}
$$

where GNSS constellations are denoted with the following superscripts: GPS $(G)$, GLONASS $(R)$, Galileo $(E)$ or BDS $(C)$ and $\mathrm{d} t_{r}^{R-G}, \mathrm{~d} t_{r}^{E-G}, \mathrm{~d} t_{r}^{C-G}$ state for GPS-GLONASS, GPS-Galileo, and GPS-BDS receiver inter system clock biases, respectively.

The vector of unknown estimates contains corrections to the a priori coordinates of the station $\left\{\delta x_{r}, \delta y_{r}, \delta z_{r}\right\}$, 
receiver clock offset $\left(\mathrm{d} \bar{t}_{r}\right)$, a set of receiver inter system clock biases $\left\{\mathrm{d} t_{r}^{R-G}, \mathrm{~d} t_{r}^{E-G}, \mathrm{~d} t_{r}^{C-G}\right\}$, a set of ambiguity parameters $\left\{A_{r}^{s, G}, A_{r}^{s, R}, A_{r}^{s, E}, A_{r}^{s, C}\right\}$, and tropospheric nonhydrostatic zenith delay $\left(\mathrm{ZWD}_{r}\right)$.

\section{Data collection}

The dataset was acquired on November 17-19, 2020 and June, 16-20, 2021 in the vicinity of UWM Olsztyn campus. GNSS measurements were collected with $5 \mathrm{~s}$ sampling interval and two collocated zero-baselines (ZB) (Fig. 1). One used two u-blox C099-F9P evaluation kits equipped with ZED F9P chipsets. An antenna splitter connected these to the same u-blox ANN-MB patch antenna having a circular ground plane. Another u-blox receiver of the same type and Trimble Alloy shared Leica LEIAR 10 antenna. The antennas were mounted on the oblique roof with some obstacles. Therefore, a non-negligible multipath effect is expected. Since the inter-antenna distance was $50 \mathrm{~cm}$, the observational conditions may be considered as the same for all receivers. The employed low-cost receivers acquire GPS L1 C/A and L2C, Galileo E1-B/C and E5b, BeiDou B1I and B2I, as well as GLONASS L1 and L2 signals. Since the SF-IF model only uses signals from the first frequency, the observations transmitted on other frequency bands have not been analyzed. During the first experiment, the Trimble receiver operated with enabled Everest ${ }^{\mathrm{TM}}$ multipath rejection technology that takes advantage of advanced digital signal processing within the satellite signal tracking loops. During the experiment conducted in June 2021, this option was disabled after two days of data collection to evaluate its potential advantages.

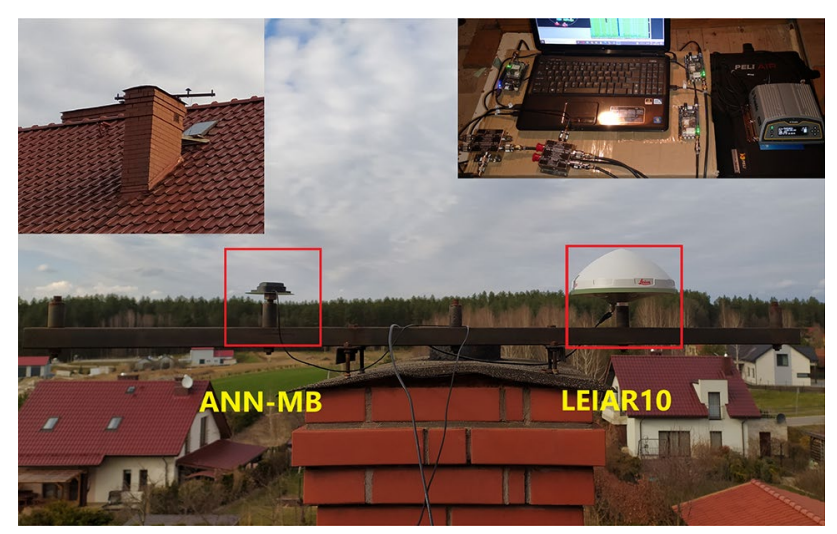

Fig. 1 Receiver and antenna setup during data collection

\section{Signal strength}

Since carrier-to-noise density ratio (C/N0) is prone to, inter alia, the losses and gains of signal strength driven by receiver hardware, this parameter is used as the first indicator that characterizes the quality of GNSS signals of lowcost receivers against high-grade ones.

Figure 2 presents the frequency distribution histograms of C/NOs for the signals on the first frequency band for the dataset collected in November 2020. The histograms show a comparable $\mathrm{C} / \mathrm{N} 0$ distribution for all receiver + antenna sets. In all cases, C/NOs exhibit the highest concentration in $41-45 \mathrm{~dB}-\mathrm{Hz}$ bin for GPS, Galileo, and GLONASS and in $36-40 \mathrm{~dB}-\mathrm{Hz}$ bin for BDS. For all constellations except $\mathrm{BDS}$, the high-grade receiver achieved the highest frequency in $41-45 \mathrm{~dB}-\mathrm{Hz}$. A significantly higher number of Galileo observations with the strongest gain that fit $46-50 \mathrm{~dB}-\mathrm{Hz}$ bin for the high-grade receiver as compared to the low-cost ones is also reported.

A more detailed view on the $\mathrm{C} / \mathrm{N} 0$ is provided by Table 1 where differences between the $\mathrm{C} / \mathrm{N} 0$ of the corresponding signals logged by Trimble Alloy + LEIAR10 and two u-blox receivers are presented. As expected, the low-cost receiver with a patch antenna always exhibits lower signal strength than the high-grade receiver. This is, for example, mirrored in between Trimble Alloy + LEIAR 10 and u-blox ZED F9P + ANN-MBaverage C/N0 differences reaching

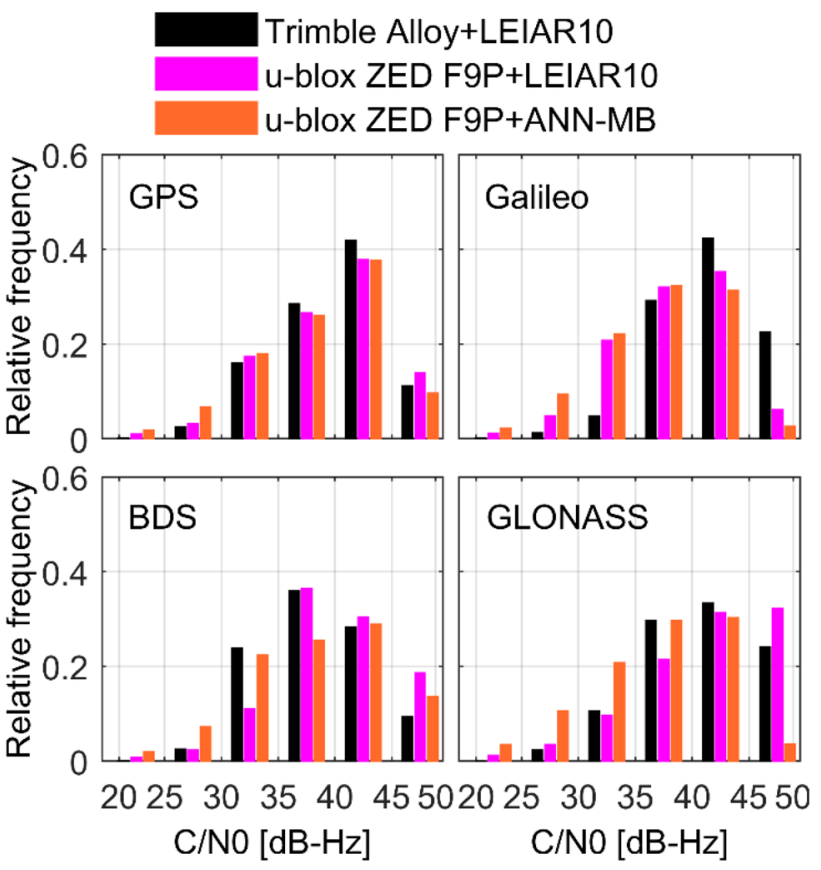

Fig. $2 \mathrm{C} / \mathrm{N} 0$ frequency distribution histograms of GNSS signals. The receiver + antenna sets are distinguished by colors 
Table 1 Average C/NO differences in $\mathrm{dB}-\mathrm{Hz}$ between corresponding GNSS signals collected by Trimble Alloy and low-cost receivers computed over all epochs and satellites

\begin{tabular}{llrlrc}
\hline Receiver & Antenna & GPS & Galileo & BDS & GLONASS \\
\hline u-blox ZED F9P & LEIAR10 & -0.5 & 2.2 & -1.2 & -2.0 \\
& ANN-MB & 0.5 & 3.7 & 0.8 & 3.1 \\
\hline
\end{tabular}
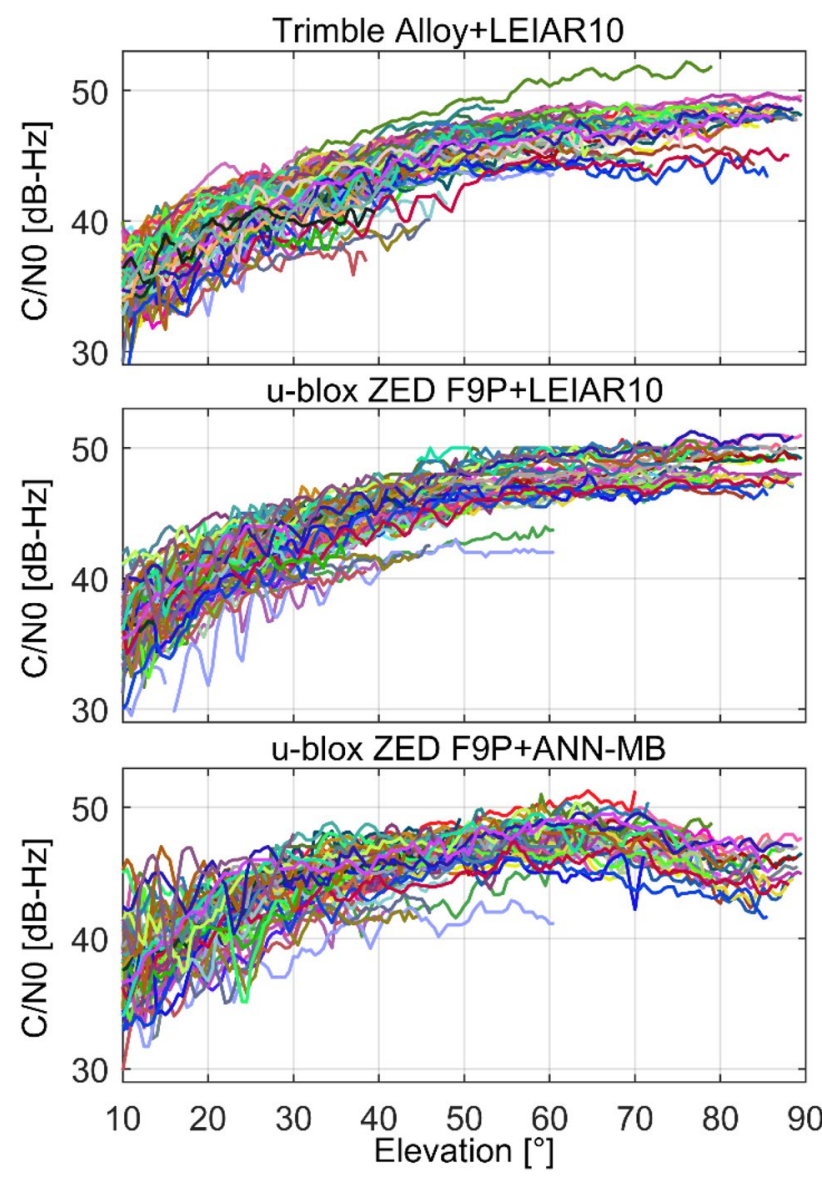

Fig. $3 \mathrm{C} / \mathrm{N} 0$ as a function of satellite elevation. $\mathrm{C} / \mathrm{N} 0$ s are averaged in $0.5^{\circ}$ bins. The values for different satellites are distinguished by colors

3.7 dB-Hz and 3.1 dB-Hz for Galileo and GLONASS observations, respectively. In the case of GPS and BDS constellations, these differences are not so significant and reach up to $0.8 \mathrm{~dB}-\mathrm{Hz}$. What is interesting, however, is that for GPS, BDS and GLONASS constellations, u-blox ZED F9P + LEIAR10 shows slightly higher values of average C/ N0 as compared to Trimble Alloy + LEIAR10. However, it is recalled that this low-cost receiver shares the survey-grade antenna with Trimble Alloy, and the latter receiver powers the shared antenna by a splitter.

What transpires from Fig. 3 is that $\mathrm{C} / \mathrm{N} 0$ of the signals collected by Trimble Alloy + LEIAR10 demonstrate a strong elevation dependence that is typical for high-grade GNSS equipment but unusual for low-cost chipsets and antennas embedded in smartphones (Li and Geng 2019; Paziewski et al. 2021, 2019). The C/N0s for ZED F9P + ANN-MB exhibit the lowest elevation dependence when compared to both receivers equipped with the LEIAR 10 antenna. The figure also shows how the patch antenna provides the observations of low-elevated satellites with C/NOs of a much noisier nature as compared to both receivers with a survey-grade antenna. Interestingly, also a low-cost receiver with a surveygrade antenna may be affected by occasional drops of the $\mathrm{C} / \mathrm{N} 0$ for low-elevated satellites. A clear decline in the $\mathrm{C} /$ $\mathrm{N} 0$ at high elevations for the low-cost receiver with a patch antenna is also worthy of notice.

\section{Observation noise}

To assess the impact of the noise and multipath effect on code observations, code-minus-carrier phase (CMC) linear combination is used, which for the satellite $s$ and receiver $r$, is expressed as follows:

$P_{r}^{s}-\Phi_{r}^{s}=M_{r, P}^{s}-M_{r, \Phi}^{s}+\epsilon_{r, P}^{s}-\epsilon_{r, \Phi}^{s}+b_{r, i}-b_{i}^{s}-B_{r, i}+B_{i}^{s}+2 I_{r}^{s}-\lambda N_{r, i}^{s}$

where $M_{r, \Phi}^{s}$ and $M_{r, P}^{s}$ denote the multipath effect in the unit of meters for phase and code observations, respectively.

CMC LC highlights the combined impact of noise and multipath of code observations but is also subject to ambiguities and satellite/receiver code and phase biases that are considered constant over time, as well as a doubled ionospheric delay that, in turn, is obviously a time-variant factor. All these unwanted effects were properly handled to isolate code noise and multipath as shown in (de Bakker et al. 2012).

Figure 4 shows the example time series of CMC LC and the corresponding C/N0 records for GPS PRN 12 that are typical for all satellites. An observer may notice that surprisingly, GPS CMC LCs for low-cost receivers demonstrate less noise manifested by a random component of the time series compared to the high-grade receiver. This finding holds true only for the signals with relatively high signal strength, i.e., $\mathrm{C} / \mathrm{N} 0$ over approximately $35 \mathrm{~dB}-\mathrm{Hz}$. On the contrary, CMC values that are characterized with low $\mathrm{C} / \mathrm{N} 0$ are much more disturbed for low-cost receivers when compared to the highgrade one and exhibit a high magnitude of the low-frequency component. Such effects are clearly seen at the beginning and at the end of the observational arcs when low-elevated signals are acquired. Such behavior of low-cost CMC time series may be attributed to the multipath effect. A less noisy nature of the random component of CMC LC time series for low-cost receivers compared to Trimble Alloy may result 

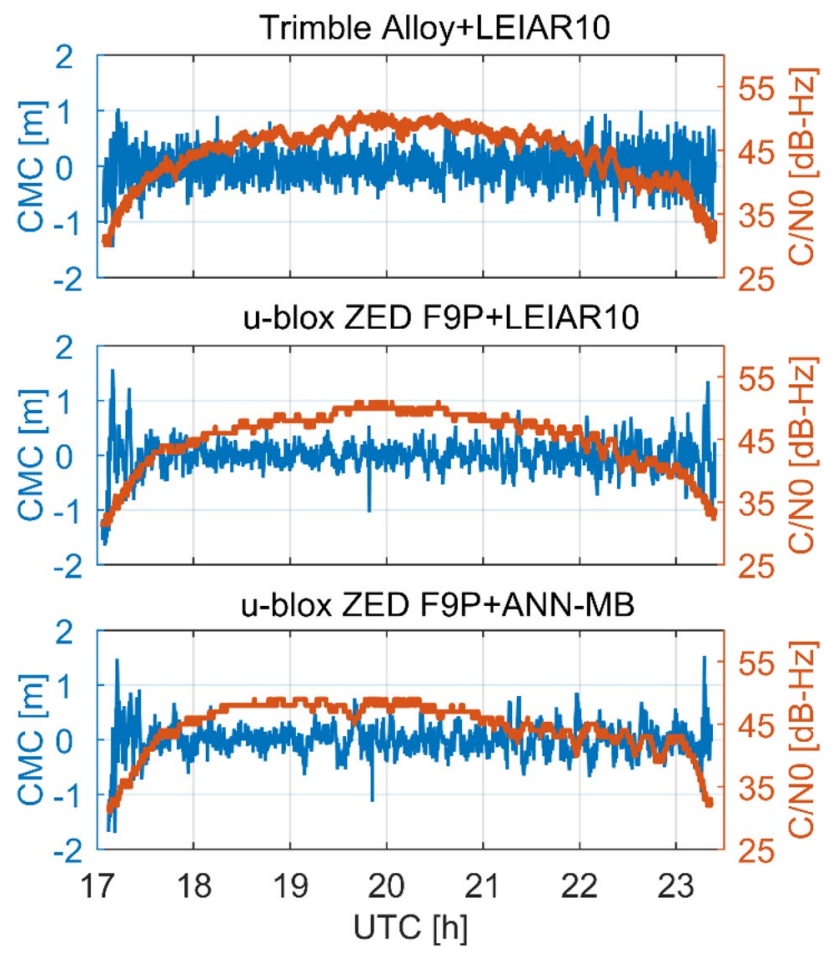

Fig. 4 CMC linear combination (in blue) and C/NO (in orange) of C1-L1 GPS PRN 12 signals on November 17, 2020

from carrier- or Doppler-smoothing of code pseudoranges (Park et al. 2017; Odolinski and Teunissen 2020). However, at this time, we are not informed about specific signal processing algorithms that are implemented in the employed low-cost receivers.

In Fig. 5, standard deviations (STD) of CMC LC as a function of signal strength are shown. The figure confirms a much noisier nature of code observations with $\mathrm{C} / \mathrm{N} 0$ lower than approximately $35 \mathrm{~dB}-\mathrm{Hz}$ for low-cost receivers compared to the high-grade one. This finding is especially true for the receiver equipped with a path antenna. The figure also reveals that code observations of the high-grade receiver are much less dependent on $\mathrm{C} / \mathrm{N} 0$ than those of low-cost equipment.

Table 2 reports CMC LC STDs that are distinguished between these related to all of the acquired observations and those related to the dataset with excluded observations characterized with low $\mathrm{C} / \mathrm{N} 0$ records (below $35 \mathrm{~dB}-\mathrm{Hz}$ ).

As expected, the CMC STDs for the low-cost receiver with a patch antenna always exhibit the highest values. What was also found is an identical performance of highgrade receivers and the low-cost receiver with a surveygrade antenna in terms of GPS L1 code observation noise expressed by CMC STD equaled to $32 \mathrm{~cm}$. Table 2 also reveals that Trimble Alloy significantly outperforms lowcost receivers in terms of Galileo E1 code accuracy. For the former receiver CMC STD equals to $25 \mathrm{~cm}$, whereas for

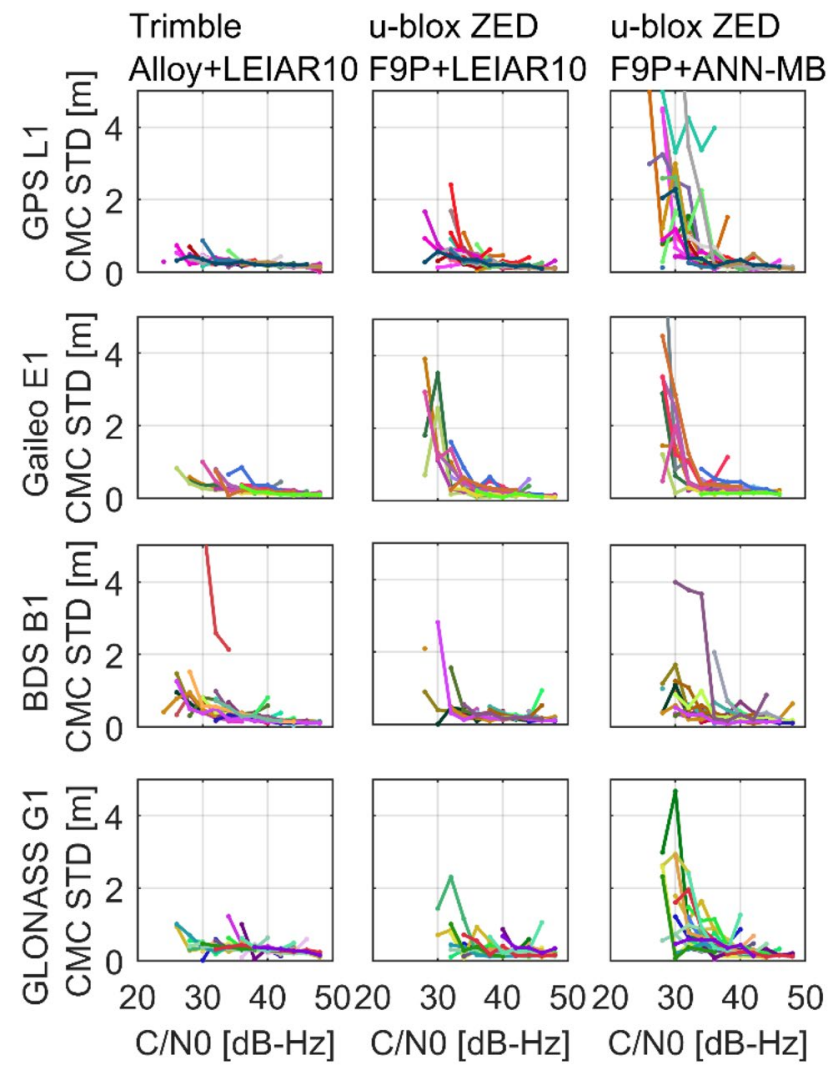

Fig. 5 STDs of CMC LC as a function of C/NO. The results corresponding to different satellites are distinguished by colors. The STDs are averaged over consecutive satellites in $2 \mathrm{~dB}-\mathrm{Hz}$ bins

low-cost receivers, these statistics reach $37 \mathrm{~cm}$ and $41 \mathrm{~cm}$, respectively. Such results are in agreement with C/NO frequency distribution histograms given in Fig. 2 where numerous Galileo observations with the highest C/N0 logged by the high-grade receiver were shown. The opposite situation occurs for code noise of BDS and GLONASS. Surprisingly, in these cases, the low-cost receiver with a survey-grade antenna outperforms high-grade equipment. However, this is consistent with a slightly higher average $\mathrm{C} / \mathrm{N} 0$ for the former receiver than the latter, reported in Table 1. One should note that BDS- 2 code observations are subject to elevation-dependent variations of code pseudoranges that may gain the CMC STDs (Wanninger and Beer 2015). Overall, the results are in line with the findings of Odolinski and Teunissen (2019), who reported a code noise fitting the range of 43-49 $\mathrm{cm}$ depending on the constellation for u-blox EVK-M8T + ANN-MS.

Further inspection of the statistics given in Table 2 reveals a noticeable reduction of CMC STDs for low-cost receivers after the elimination of low-gain signals. Such high enhancement is not observed for the high-grade receiver, which was however expected if we recall the results of CMC STD as a function of $\mathrm{C} / \mathrm{N} 0$ illustrated in Fig. 5. Taking as an 
Table 2 CMC LC STDs in the units of centimeters are computed as a mean over all epochs and satellites

\begin{tabular}{|c|c|c|c|c|c|c|c|c|c|}
\hline \multirow[t]{2}{*}{ Receiver } & \multirow[t]{2}{*}{ Antenna } & \multicolumn{2}{|l|}{ GPS L1 } & \multicolumn{2}{|c|}{ Galileo E1 } & \multicolumn{2}{|l|}{ BDS B1 } & \multicolumn{2}{|c|}{ GLONASS G1 } \\
\hline & & $\begin{array}{l}\text { All } \\
\text { observa- } \\
\text { tions }\end{array}$ & $\begin{array}{l}\mathrm{C} / \\
\mathrm{N} 0>35 \mathrm{~dB}-\mathrm{Hz}\end{array}$ & $\begin{array}{l}\text { All } \\
\text { observa- } \\
\text { tions }\end{array}$ & $\begin{array}{l}\mathrm{C} / \\
\mathrm{N} 0>35 \mathrm{~dB}-\mathrm{Hz}\end{array}$ & $\begin{array}{l}\text { All } \\
\text { observa- } \\
\text { tions }\end{array}$ & $\begin{array}{l}\mathrm{C} / \\
\mathrm{N} 0>35 \mathrm{~dB}-\mathrm{Hz}\end{array}$ & $\begin{array}{l}\text { All } \\
\text { observa- } \\
\text { tions }\end{array}$ & $\begin{array}{l}\mathrm{C} / \\
\mathrm{N} 0>35 \mathrm{~dB}-\mathrm{Hz}\end{array}$ \\
\hline Trimble alloy & LEIAR10 & 32 & 31 & 25 & 24 & 35 & 30 & 44 & 43 \\
\hline \multirow[t]{2}{*}{ ZED F9P } & LEIAR10 & 32 & 28 & 37 & 34 & 32 & 30 & 34 & 33 \\
\hline & ANN-MB & 39 & 31 & 41 & 36 & 42 & 40 & 48 & 44 \\
\hline
\end{tabular}

Table 3 STDs computed over all epochs and satellites and normalized to the undifferenced level for u-blox ZED F9P zero-baseline double differenced observations

\begin{tabular}{lllll}
\hline Observation & GPS L1 & Galileo E1 & BDS B1 & GLONASS G1 \\
\hline Phase $(\mathrm{mm})$ & 2.9 & 2.8 & 2.5 & 4.1 \\
Code $(\mathrm{cm})$ & 29 & 32 & 32 & 35 \\
\hline
\end{tabular}

example the GPS L1 observations, after the elimination of low-gain signals, Trimble Alloy CMC STD improved only by a $1 \mathrm{~cm}$, whereas for the low-cost receivers with a surveygrade antenna and a patch antenna, these improvements reached $4 \mathrm{~cm}$ and $8 \mathrm{~cm}$, respectively. The outcomes confirm that low-cost GNSS equipment is highly prone to the multipath effect that mostly affects low-elevated and therefore low-gain signals, and may exhibit a competitive performance to that of high-grade grade equipment providing that multipath affected observations were eliminated or corrected.

\section{Phase and code noise analysis with zero-baseline setup}

Double-differenced (DD) observations of zero-baseline (ZB) setup built of two ZED F9P receivers connected by a splitter to the same ANN-MB patch antenna are used to assess the noise of phase observations.

Phase STDs of low-cost receivers that were normalized to the undifferenced level are presented in Table 3. The results show a precision of phase observations comparable to highgrade receivers and an agreement with the findings of Odolinski and Teunissen (2019). For all except the GLONASS constellation, the phase STD did not exceed $2.9 \mathrm{~mm}$. The noise of GLONASS phase observations is higher and equals to $4.1 \mathrm{~mm}$. Nonetheless, the noise of phase observations has a marginal impact on SF-IF PPP performance since it is approximately two orders lower as compared to that of code observations.

More importantly, the ZB setup allows the separation of the observational noise from the multipath effect since the latter affects the observations of both receivers of the $\mathrm{ZB}$ setup in the same manner and thus is reduced in double-differencing. The results given in Table 3 confirm a high impact of multipath on low-cost equipment since STDs of code noise are noticeably lower as compared to those reported in Table 2, which correspond to the combined impact of noise and multipath. U-blox ZED F9P + ANN-MB code STDs reduce from $39 \mathrm{~cm}, 41 \mathrm{~cm}, 42 \mathrm{~cm}$, and $48 \mathrm{~cm}$ for GPS, Galileo, BDS, and GLONASS to $29 \mathrm{~cm}, 32 \mathrm{~cm}, 32 \mathrm{~cm}$, and $35 \mathrm{~cm}$, respectively, when the multipath effect has been eliminated in ZB setup.

\section{Performance assessment of SF-IF PPP with low-cost receivers}

In this section, the details of the processing strategy are provided and the positioning performance assessment of quadruple-constellation SF-IF PPP is presented. A full dataset was processed using processing windows of different lengths.

\section{Processing strategy}

Correction models that are typically considered in the multiconstellation PPP were employed. Specifically final MGEX orbits and clock corrections were used (Prange et al. 2020). The MGEX bias products are applied to account for satellite code biases and to correct satellite clock offsets containing ionospheric-free satellite code hardware delays for singlefrequency measurements. A priori hydrostatic tropospheric delays are modeled using the Saastamoinen model and Global Mapping Function (Boehm et al. 2006; Saastamoinen 1973). Other corrections to eliminate effects such as relativity, solid earth tides, phase wind-up effect, and antenna phase center offset and variations with igs14.atx files are also accounted for according to the conventional models (Kouba and Héroux 2001).

The parameter estimation is performed using the Kalman filter. Spectral density values of process noise equal to $10^{2} \mathrm{~m}^{2} \mathrm{~s}^{-1}, 10^{-7} \mathrm{~m}^{2} \mathrm{~s}^{-1}$, and $10^{-9} \mathrm{~m}^{2} \mathrm{~s}^{-1}$ for receiver clock offset, receiver inter system clock biases, and nonhydrostatic zenith tropospheric delays, respectively, were adopted in random walk processes. Static coordinates and 
float ambiguity parameters are considered as constant values. The zenith-referenced STDs of code observations are adopted, and taking advantage of the observation assessment results, an elevation-dependent weighting scheme based on the cosecant function is used. The data were processed with 5 s sampling interval and a cutoff angle of 10 degrees.

\section{Positioning results}

The performance of quadruple-constellation SF-IF PPP was evaluated in terms of positioning errors and convergence time. Positioning errors are computed by referring the coordinate estimates to the benchmark coordinates. Convergence time is defined here as the period required to achieve and keep a position for at least 5 min with a three-dimensional (3D) error lower than the adopted thresholds of $1 \mathrm{~m}$ or $1.5 \mathrm{~m}$.

Figure 6 shows a snapshot positioning performance of low-cost receivers against a high-grade receiver. The figure presents example time series of quadruple-constellation SF-IF PPP positioning errors for static daily solutions on November 17-18, 2020. What transpires from the figure is a noticeably slower convergence for both low-cost receivers when compared to the high-grade one. A clear benefit from the application of survey-grade antenna instead of the
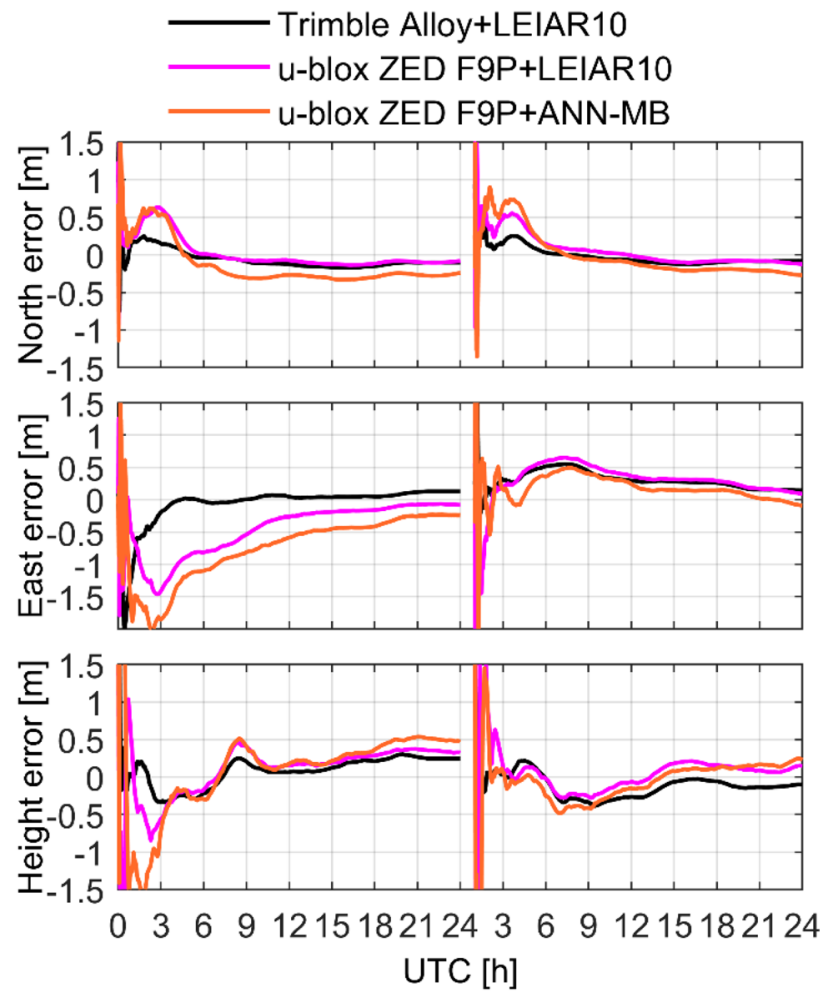

Fig. 6 Time series of positioning errors for SF-IF PPP static daily solutions on November 17-18, 2020. A gap 0-1 UTC on November 18,2020 is caused by an interruption in data collection. Trimble receiver operates with enabled Everest ${ }^{\mathrm{TM}}$ algorithm patch one is also reported, as u-blox ZED F9P + LEIAR10 gives lower positioning errors and faster convergence when compared to ZED F9P + ANN-MB.

Figure 7 presents SF-IF PPP 3D positioning root-meansquare (RMS) errors that were averaged over the full dataset. The Trimble receiver results are distinguished between those that are related to the period with an enabled Everest $^{\mathrm{TM}}$ multipath rejection technology and those that refer to the period after disabling the function. The figure shows how the 3D positioning accuracy converges to the level of $0.5 \mathrm{~m}$ after approximately $4-5 \mathrm{~h}$ depending on the employed receiver + antenna set. The full dataset results confirm the snapshot of daily solutions given in Fig. 6, as Trimble Alloy converges noticeably faster than both low-cost receivers. A convergence enhancement from the application of the Everest $^{\mathrm{TM}}$ algorithm in the Trimble receiver is worthy of attention.

A detailed evaluation of the positioning results begins with the convergence time assessment. Figure 8 provides a focus on the average over the full dataset 3D RMS positioning errors that were obtained when the filter was reinitialized every $1 \mathrm{~h}$. The most evident outperformance of high-grade receivers over the low-cost ones is observed during the first $45 \mathrm{~min}$ of data processing. Suppose we focus on the first 15-20 min. In that case, we will also notice the benefit of applying the survey-grade antenna to low-cost receiver positioning and the advantage from Trimble's Everest ${ }^{\mathrm{TM}}$ technology. Nonetheless, after approximately a $1 \mathrm{~h}$ initialization period, all receivers reach a comparable accuracy level.

Table 4 summarizes the performance of the convergence time. Statistics prove how the high-grade receiver converges faster than the low-cost ones. Trimble Alloy with enabled Everest $^{\mathrm{TM}}$ requires $20.8 \mathrm{~min}$ to converge to the defined 3D accuracy threshold of $1 \mathrm{~m}$. The corresponding indicators for ZED F9Ps with a patch or a survey-grade antenna are

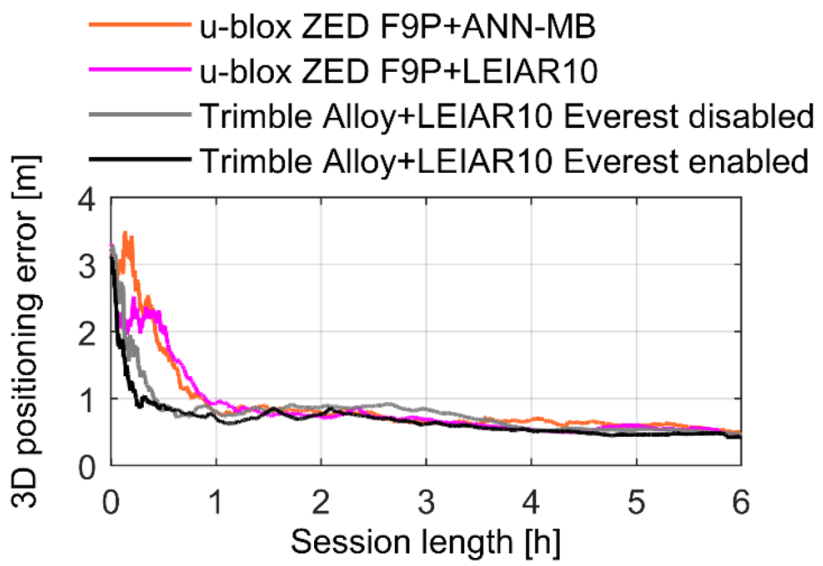

Fig. 7 Time series of 3D positioning RMS errors of SF-IF PPP. The solutions obtained using a filter reinitialization every $6 \mathrm{~h}$ were averaged over the full dataset 


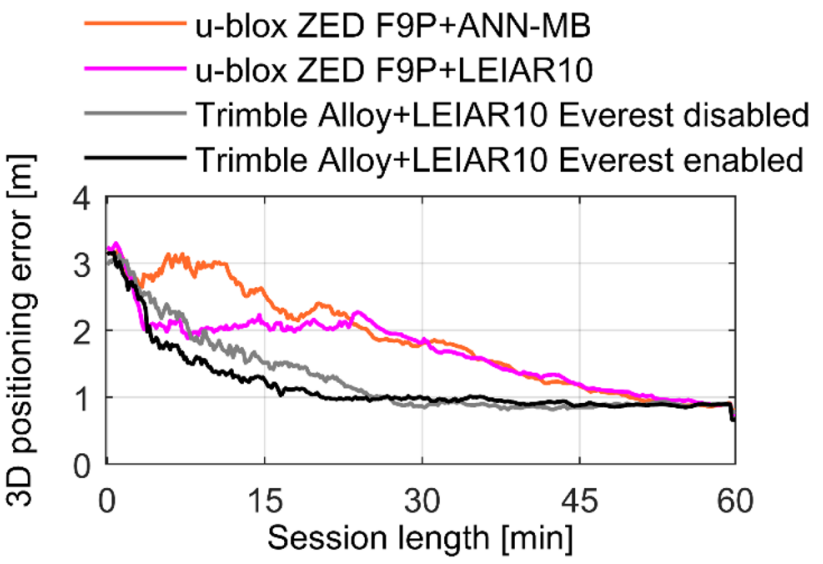

Fig. 8 Time series of 3D positioning RMS errors of SF-IF PPP. The solutions obtained using a filter reinitialization every $1 \mathrm{~h}$ were averaged over the full dataset

more than twice as high. An advantage from the Everest $\mathrm{t}^{\mathrm{TM}}$ algorithm is also proved. The convergence time extends for almost one-quarter from 20.8 up to $25.7 \mathrm{~min}$, when this option is disabled in the Trimble receiver.

The statistics for a less challenging 3D positioning error threshold of $1.5 \mathrm{~m}$ aim to highlight the divergences between the receivers during the filter initialization period. Trimble Alloy with Everest ${ }^{\mathrm{TM}}$ is the best performing receiver, whereas ZED F9P with a patch antenna is the worst one. The latter needs $37.8 \mathrm{~min}$ to reach the defined $3 \mathrm{D}$ positioning accuracy level, whereas it is only $8.3 \mathrm{~min}$ for the former.

To further investigate the inequalities in the positioning performance among the employed receivers, the empirical RMSs of positioning errors are presented in Table 5. The statistics correspond to the accuracy level that may be reached after $15 \mathrm{~min}, 45 \mathrm{~min}$, and $6 \mathrm{~h}$ of data processing. For the first two intervals, the positioning accuracy is expected to be significantly lower than that of daily solutions. In the latter case, the positioning accuracy may converge to subdecimeter level, as shown in Fig. 6 and past studies (Cai et al. 2013). However, such long site occupations are rarely expected in real-life applications.

Overall, Table 5 shows that the positioning errors are at a dm-level depending on the adopted processing window and receiver. As we may read from the table, Trimble Alloy with Everest ${ }^{\mathrm{TM}}$ always outperforms both low-cost receiver sets and Trimble Alloy with a disabled Everest ${ }^{\mathrm{TM}}$ in terms of positioning accuracy. The Trimble Alloy performance corresponds to the previous results reported for high-grade receivers in Bahadur and Nohutcu (2021), which showed an 3D RMS of 0.5-0.7 m depending on the satellite products used, and also confirmed the better performance of multiconstellations solution over single-, dual- or triple-constellation solutions.

Moreover, the statistics manifest significant divergences in positioning performance between high-grade and lowcost receivers during the initialization period. Taking $15 \mathrm{~min}$ long processing window as an example, averaged RMSs of positioning errors for Trimble Alloy with enabled Everest ${ }^{\mathrm{TM}}$ are of $47.1 \mathrm{~cm}, 68.7 \mathrm{~cm}$, and $49.0 \mathrm{~cm}$ for North, East, and Up components, respectively. As expected, a lower accuracy is reached by the Trimble Alloy with the disabled Everest $^{\mathrm{TM}}$ and in this case RMSs are of $45.0 \mathrm{~cm}, 76.6 \mathrm{~cm}$, and $64.0 \mathrm{~cm}$, for the respective coordinate components. ZED F9P + LEIAR10 antenna gives comparable to the high-grade receiver RMSs for North and East components of $46.0 \mathrm{~cm}$, $73.4 \mathrm{~cm}$, respectively, but over three times higher RMS for the vertical one, that is of $190.8 \mathrm{~cm}$. This between-receiver divergence reaches an even higher magnitude when the high-grade equipment is compared to the low-cost receiver
Table 4 Convergence time of SF-IF PPP. The statistics that were computed individually for every $1 \mathrm{~h}$ window solution are averaged over the full dataset

\begin{tabular}{lllll}
\hline Receiver & $\begin{array}{l}\text { Multipath mitigation } \\
\text { algorithm }\end{array}$ & Antenna & \multicolumn{2}{l}{ Convergence time (min) } \\
\cline { 4 - 5 } & & & 3D error $<1.5 \mathrm{~m}$ & 3D error $<1 \mathrm{~m}$ \\
\hline Trimble alloy & Enabled & LEIAR10 & 8.3 & 20.8 \\
& Disabled & & 15.7 & 25.7 \\
ZED F9P & Not applicable & & 36.8 & 52.2 \\
& & ANN-MB & 37.8 & 50.0 \\
\hline
\end{tabular}

Table 5 Empirical positioning RMSs of SF-IF PPP computed over the full dataset

\begin{tabular}{|c|c|c|c|c|c|c|c|c|c|c|c|}
\hline \multirow[t]{2}{*}{ Receiver } & \multirow{2}{*}{$\begin{array}{l}\text { Multipath miti- } \\
\text { gation algorithm }\end{array}$} & \multirow[t]{2}{*}{ Antenna } & \multicolumn{3}{|c|}{ After $15 \mathrm{~min}$} & \multicolumn{3}{|c|}{ After 45 min } & \multicolumn{3}{|c|}{ After $6 \mathrm{~h}$} \\
\hline & & & $N(\mathrm{~cm})$ & $E(\mathrm{~cm})$ & $U(\mathrm{~cm})$ & $N(\mathrm{~cm})$ & $E(\mathrm{~cm})$ & $U(\mathrm{~cm})$ & $N(\mathrm{~cm})$ & $E(\mathrm{~cm})$ & $U(\mathrm{~cm})$ \\
\hline \multirow[t]{2}{*}{ Trimble alloy } & Enabled & LEIAR10 & 47.1 & 68.7 & 49.0 & 27.7 & 43.1 & 50.6 & 9.8 & 20.8 & 23.3 \\
\hline & Disabled & & 45.0 & 76.6 & 64.0 & 28.0 & 43.7 & 44.6 & 8.8 & 23.5 & 30.5 \\
\hline \multirow[t]{2}{*}{ ZED F9P } & Not applicable & & 46.0 & 73.4 & 190.8 & 25.7 & 37.7 & 89.3 & 9.6 & 22.9 & 27.8 \\
\hline & & ANN-MB & 54.2 & 115.5 & 172.9 & 33.0 & 46.4 & 77.4 & 14.4 & 26.1 & 23.9 \\
\hline
\end{tabular}


with a patch antenna. Such outcomes prove how the multipath effect, to which the low-cost receivers are especially prone, propagates into estimates and, as a consequence, produces biased position time series and deteriorates position convergence.

Inspecting Table 5 again, the reader may notice that after a few hours, the position converges to the $3 \mathrm{D}$ positioning RMS of about 3-4 dm for all receivers and therefore, the important disagreements between the receivers disappear.

\section{Insight into the observation residuals}

SF-IF observation residuals are examined to understand the poorer positioning performance of low-cost receivers when compared to the high-grade ones. An insight into the example residuals of GPS PRN 12 is given as they are typical for all satellites. Figure 9 shows the time series of residuals in gray color for June 16-17, 2021 and satellite elevation in a blue color when the Trimble receiver operated with enabled Everest ${ }^{\mathrm{TM}}$ algorithm. The residual time series demonstrate two components, a high-frequency random component that is most likely driven only by observation noise and a lowfrequency one related to unmodeled errors that remain in the SF-IF observations, which is predominately the multipath effect (Sterle et al. 2015). The red line denotes the residuals that were filtered with a moving average to expose the lowfrequency component. The STDs given in Fig. 9 are defined from the low-frequency component of the residuals.

The hypothesis that the multipath effect is mainly responsible for the poor performance of low-cost receiver positioning may be verified by an analysis of day-by-day repeatability of the residual time series. It is evident that the multipath effect and antenna phase-center variation error exhibit repeatability since the same measuring environment is provided each sidereal day by the repetition of GPS orbits (Xue et al. 2021). Indeed, a consistency between the low-frequency components of the residuals of consecutive days for the low-cost receivers that are given in Fig. 9 is discovered.

A high impact of the multipath is also confirmed by the change in the nature of the Trimble residuals after the deactivation of the Everest ${ }^{\mathrm{TM}}$ multipath rejection algorithm. Such residuals, shown in Fig. 10, are subject to evident low-frequency components characterized with STDs of $0.13-0.14 \mathrm{~m}$ that are about $60 \%$ higher as compared to the period when the Everest ${ }^{\mathrm{TM}}$ was enabled (c.f. Figure 9). A noticeable drop in STD of the filtered residuals driven by the multipath mitigation algorithm implies that there is still some room for positioning improvement, providing that the multipath is carefully handled.

It also follows from Fig. 9 that the estimated observation residuals for both low-cost receivers exhibit a lower noise that is represented by a random component of the time series and a higher impact of low-frequency effects that is
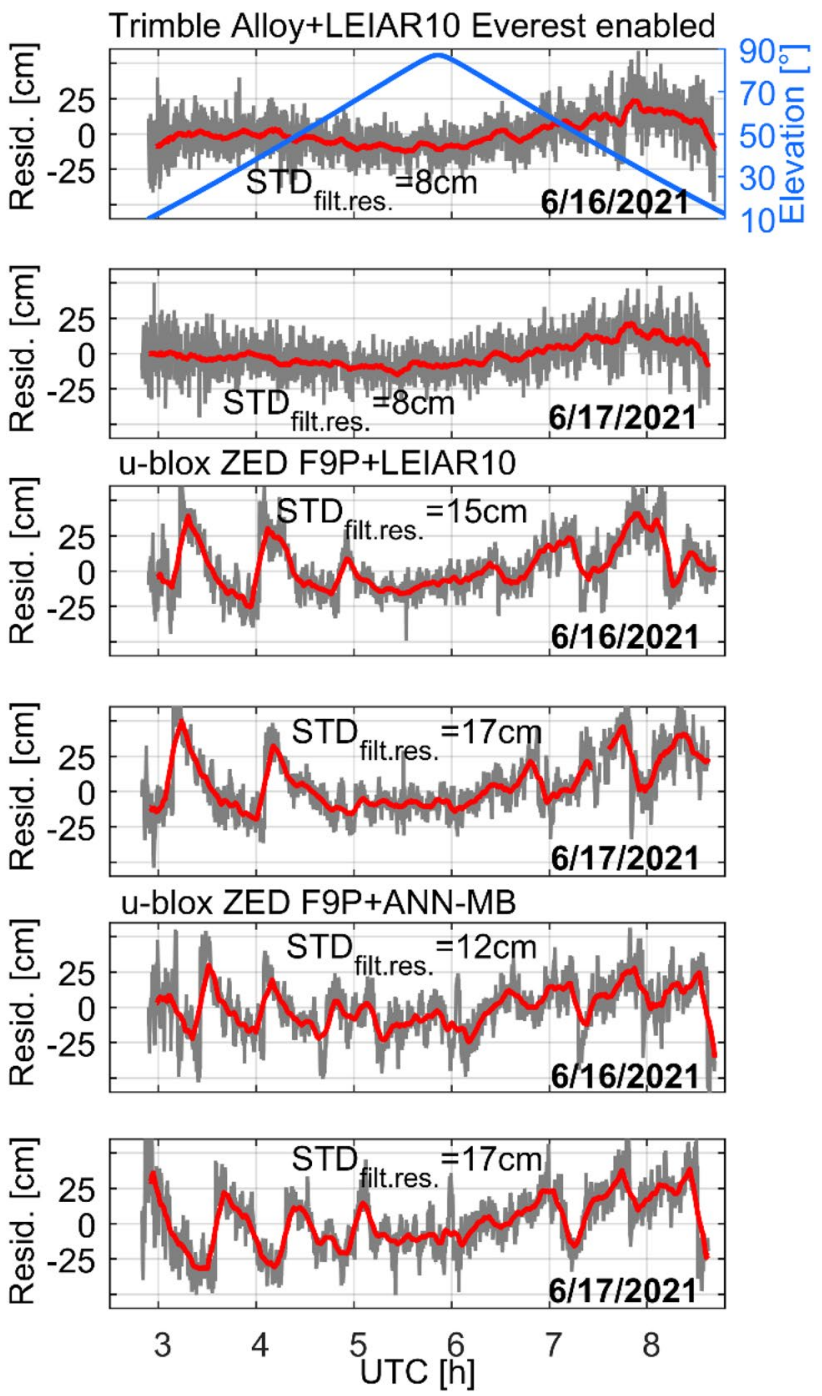

Fig. 9 An example of SF-IF PPP observation residuals time series in gray for GPS PRN 12 obtained on June 16-17, 2021. Red lines represent the residuals filtered with a moving average filter and corresponded to low-frequency unmodeled effects. Satellite elevation is shown in blue. The first and second panels correspond to the residuals for Trimble Alloy + LEIAR10 with enabled Everest ${ }^{\mathrm{TM}}$. The U-blox ZED F9P + LEIAR10 residuals are given in panels three and four, respectively. ZED F9P + ANN-MB residuals are shown in fifth and sixth panels, correspondingly

likely driven by multipath as compared to high-grade receivers. Such results are consistent with code-minus-carrier LC given in Fig. 4.

If we further investigate Fig. 9, we discover that an application of the survey-grade antenna to the low-cost receiver did not significantly change the nature of residual time series as they still exhibit a noticeable low-frequency component. This finding is supported by the example STDs of SF-IF residuals given in the figure, as they are always higher than the Trimble receiver. Therefore, the low-frequency component of the residual time series is attributed to the way how 

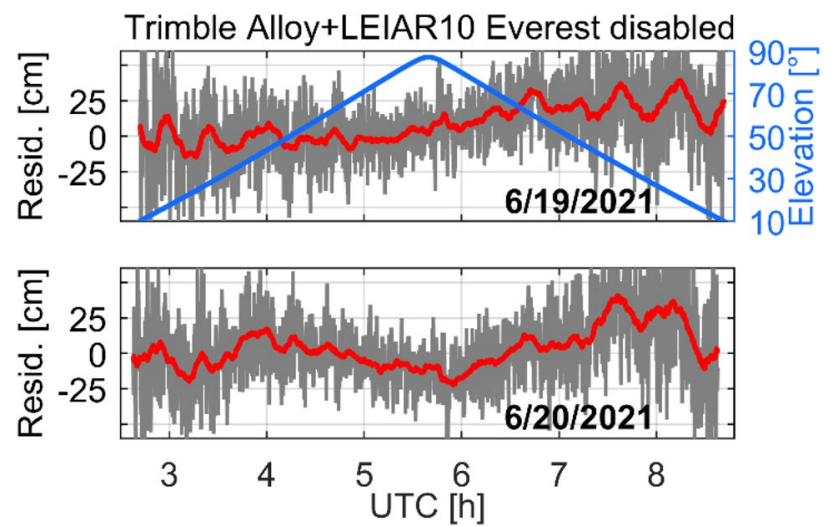

Fig. 10 SF-IF PPP observation residuals time series for GPS PRN 12 obtained on June 19-20, 2021 for Trimble Alloy + LEIAR10 with disabled Everest ${ }^{\mathrm{TM}}$

receivers handle the multipath. It may be concluded that the application of a survey-grade antenna instead of a patch one, enhances the PPP performance of low-cost receivers to some extent that is in agreement with the past studies on RTK (Odolinski and Teunissen 2016). However, it requires advanced multipath mitigation algorithms to reach the positioning performance close to that of high-grade receivers. Given that theoretically, phase multipath may reach only up to a quarter of a signal wavelength, it becomes clear that the multipath of code observations should be a subject of further investigations with regard to the low-cost receiver SF-PPP.

\section{Conclusions}

First, it was shown that the low-cost receiver with a patch antenna exhibits lower records of the C/N0 than the highgrade receiver. Then, a weaker elevation dependence of $\mathrm{C} / \mathrm{N} 0$ for a patch antenna as compared to the high-grade one was proved. The results also showed how the low-cost antenna provides the observations of low-elevated satellites with $\mathrm{C} / \mathrm{N} 0$ s of much noisier nature than the receivers with a survey-grade antenna and exhibits a drop in $\mathrm{C} / \mathrm{N} 0$ at high elevations.

The observation assessment revealed that low-cost receivers may offer code measurements characterized with a similar noise compared to high-grade receivers providing that the multipath effect has been eliminated. A slight outperformance of the low-cost receivers over the high-grade one in terms of code noise was noticed for GPS and GLONASS constellations when low-gain signals were eliminated. Moreover, the ZB setup experiment proved a high precision of low-cost receiver phase observations corresponding to high-grade receivers.

Assessing the positioning performance in a non-negligible multipath environment, it was then demonstrated how
SF-IF PPP for the high-grade receiver converges approximately two times faster than for the low-cost receiver with a patch antenna. Analysis of the positioning errors confirmed a significant outperformance of the high-grade equipment when compared to the low-cost one during the initialization period. The tests demonstrated how after approximately $6 \mathrm{~h}$ the low-cost receivers may reach a comparable to highgrade ones accuracy level characterized with 3D RMS of 0.3-0.4 m. It was also shown how the application of a survey-grade antenna instead of a patch one might enhance the low-cost receiver positioning by improving the convergence time and reducing positioning errors.

Finally, SF-IF observation residuals were investigated to understand the poorer positioning performance of the lowcost receivers when compared to the high-grade ones. It was revealed that the multipath effect is a significant part of the observation residuals and a dominant factor that constrains the performance of SF-IF PPP with low-cost receivers. It is anticipated that an application of advanced multipath mitigation algorithms similar to Trimble's Everest ${ }^{\mathrm{TM}}$ may move the positioning performance of low-cost receivers towards the level close to that of high-grade receivers.

Acknowledgements This contribution was supported by the National Science Centre, Poland: Project No. 2016/23/D/ST10/01546.

Data availability The GNSS observational data can be made available upon request by contacting the author.

Open Access This article is licensed under a Creative Commons Attribution 4.0 International License, which permits use, sharing, adaptation, distribution and reproduction in any medium or format, as long as you give appropriate credit to the original author(s) and the source, provide a link to the Creative Commons licence, and indicate if changes were made. The images or other third party material in this article are included in the article's Creative Commons licence, unless indicated otherwise in a credit line to the material. If material is not included in the article's Creative Commons licence and your intended use is not permitted by statutory regulation or exceeds the permitted use, you will need to obtain permission directly from the copyright holder. To view a copy of this licence, visit http://creativecommons.org/licenses/by/4.0/.

\section{References}

Bahadur B, Nohutcu M (2020) Galileo-based precise point positioning with different MGEX products. Meas Sci Technol 31:094009. https://doi.org/10.1088/1361-6501/ab8b85

Bahadur B, Nohutcu M (2021) Real-time single-frequency multiGNSS positioning with ultra-rapid products. Meas Sci Technol 32:014003. https://doi.org/10.1088/1361-6501/abab22

Boehm J, Niell A, Tregoning P, Schuh H (2006) Global Mapping Function (GMF): a new empirical mapping function based on numerical weather model data. Geophys Res Lett 33(7):L07304. https:// doi.org/10.1029/2005GL025546

Cai C, Liu Z, Luo X (2013) Single-frequency ionosphere-free precise point positioning using combined GPS and GLONASS 
observations. J Navig 66(3):417-434. https://doi.org/10.1017/ S0373463313000039

de Bakker PF, Tiberius CCJM, van der Marel H, van Bree RJP (2012) Short and zero baseline analysis of GPS L1 C/A, L5Q, GIOVE E1B, and E5aQ signals. GPS Solut 16:53-64. https://doi.org/10. 1007/s10291-011-0202-3

Garrido-Carretero MS, de Lacy-Pérez de los Cobos MC, BorqueArancón MJ, Ruiz-Armenteros AM, Moreno-Guerrero R, GilCruz AJ (2019) Low-cost GNSS receiver in RTK positioning under the standard ISO-17123-8: a feasible option in geomatics. Measurement 137:168-178. https://doi.org/10.1016/j.measu rement.2019.01.045

Gill M, Bisnath S, Aggrey J, Seepersad G (2017) Precise point positioning (PPP) using low-cost and ultra-low-cost GNSS receivers. In: Proceedings of ION ITM GNSS+ 2017, Institute of Navigation, Portland, Oregon, September 25-29, pp 226-236. https://doi. org/10.33012/2017.15123

Kouba J, Héroux P (2001) Precise point positioning using IGS orbit and clock products. GPS Solut 5(2):12-28. https://doi.org/10.1007/ PL00012883

Li G, Geng J (2019) Characteristics of raw multi-GNSS measurement error from Google Android smart devices. GPS Solut 23:90. https://doi.org/10.1007/s10291-019-0885-4

Li B, Zang N, Ge H, Shen Y (2019) Single-frequency PPP models: analytical and numerical comparison. J Geod 93:2499-2514. https:// doi.org/10.1007/s00190-019-01311-4

Nie Z, Liu F, Gao Y (2019) Real-time precise point positioning with a low-cost dual-frequency GNSS device. GPS Solut 24:9. https:// doi.org/10.1007/s10291-019-0922-3

Odolinski R, Teunissen PJG (2016) Single-frequency, dual-GNSS versus dual-frequency, single-GNSS: a low-cost and high-grade receivers GPS-BDS RTK analysis. J Geod 90:1255-1278. https:// doi.org/10.1007/s00190-016-0921-x

Odolinski R, Teunissen PJG (2017) Low-cost, high-precision, singlefrequency GPS-BDS RTK positioning. GPS Solut 21:1315-1330. https://doi.org/10.1007/s10291-017-0613-x

Odolinski R, Teunissen PJG (2019) An assessment of smartphone and low-cost multi-GNSS single-frequency RTK positioning for low, medium and high ionospheric disturbance periods. J Geod 93:701-722. https://doi.org/10.1007/s00190-018-1192-5

Odolinski R, Teunissen PJG (2020) Best integer equivariant estimation: performance analysis using real data collected by low-cost, single- and dual-frequency, multi-GNSS receivers for short- to long-baseline RTK positioning. J Geod 94:91. https://doi.org/10. 1007/s00190-020-01423-2

Park B, Lim C, Yun Y, Kim E, Kee C (2017) Optimal divergence-free Hatch filter for GNSS single-frequency measurement. Sensors 17(3):448. https://doi.org/10.3390/s17030448

Paziewski J, Sieradzki R, Baryla R (2019) Signal characterization and assessment of code GNSS positioning with low-power consumption smartphones. GPS Solut 23:98. https://doi.org/10.1007/ s10291-019-0892-5

Paziewski J, Fortunato M, Mazzoni A, Odolinski R (2021) An analysis of multi-GNSS observations tracked by recent Android smartphones and smartphone-only relative positioning results. Measurement 175:109162. https://doi.org/10.1016/j.measurement. 2021.109162

Prange L, Villiger A, Sidorov D, Schaer S, Beutler G, Dach R, Jäggi A (2020) Overview of CODE's MGEX solution with the focus on Galileo. Adv Space Res 66(12):2786-2798. https://doi.org/10. 1016/j.asr.2020.04.038

Saastamoinen J (1973) Contributions to the theory of atmospheric refraction: part II. Refraction corrections in satellite geodesy. Bull Geodesique 107:13-34. https://doi.org/10.1007/BF02522083

Sterle O, Stopar B, Pavlovčič Prešeren P (2015) Single-frequency precise point positioning: an analytical approach. J Geod 89:793-810. https://doi.org/10.1007/s00190-015-0816-2

Wang A, Chen J, Zhang Y, Meng L, Wang B, Wang J (2020) Evaluating the impact of CNES real-time ionospheric products on multiGNSS single-frequency positioning using the IGS real-time service. Adv Space Res 66(11):2516-2527. https://doi.org/10.1016/j. asr.2020.09.010

Wang L, Li Z, Wang N, Wang Z (2021) Real-time GNSS precise point positioning for low-cost smart devices. GPS Solut 25:69. https:// doi.org/10.1007/s10291-021-01106-1

Wanninger L, Beer S (2015) BeiDou satellite-induced code pseudorange variations: diagnosis and therapy. GPS Solut 19:639-648. https://doi.org/10.1007/s10291-014-0423-3

Wisniewski B, Bruniecki K, Moszynski M (2013) Evaluation of RTKLIB's positioning accuracy using low-cost GNSS receiver and ASG-EUPOS. TRANSNAV Int J Mar Navig Saf Sea Transp 7(1):79-85. https://doi.org/10.12716/1001.07.01.10

Xue C, Psimoulis P, Zhang Q, Meng X (2021) Analysis of the performance of closely spaced low-cost multi-GNSS receivers. Appl Geomat 13:415-435. https://doi.org/10.1007/s12518-021-00361-8

Yunck T (1996) Orbit determination. In: Parkinson BW, Spilker JJ (eds) Global positioning system-theory and applications. AIAA, Washington, D.C.

Publisher's Note Springer Nature remains neutral with regard to jurisdictional claims in published maps and institutional affiliations.

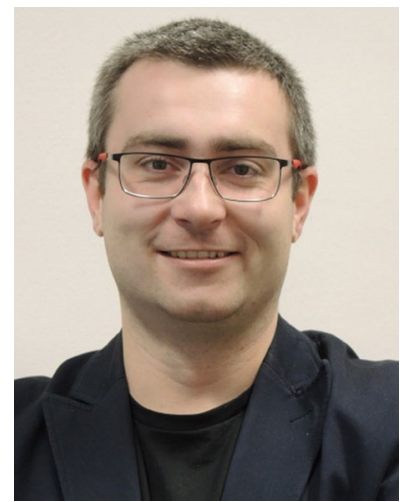

Jacek Paziewski is an associate professor at the University of Warmia and Mazury in Olsztyn. His research interests include developing multi-GNSS positioning algorithms, modeling the ionospheric delays, smartphone and low-cost receiver positioning, and high-rate data processing. 